\title{
Correlation of quantitative computed tomography derived bone density values with Hounsfield units of a contrast medium computed tomography in 98 thoraco-lumbar vertebral bodies
}

\author{
Fabian Buenger $^{1} \cdot$ Yasser Sakr $^{2} \cdot$ Niklas Eckardt $^{3} \cdot$ Christian Senft $^{1} \cdot$ Falko Schwarz $^{1}$ (D)
}

Received: 17 May 2021 / Accepted: 15 September 2021 / Published online: 25 September 2021

(c) The Author(s) 2021

\begin{abstract}
Introduction Vertebral fractures in patients with bone density reduction are often a major challenge for the surgeon, as reduced bone density can lead to screw loosening. Several options are available to determine bone density preoperatively. In our study, we investigated the correlation of Hounsfield units (HU) of a contrast medium computed tomography (CT) to the bone density values of a quantitative computed tomography (QCT) and computed a formula to estimate bone density values using $\mathrm{HU}$.

Materials and methods In our retrospective data analysis, we examine 98 vertebral bodies from 35 patients who received a contrast medium CT of the spine and a QCT, performed no longer than 1 month apart. The determined HU from the contrast medium CT were compared with the bone density values of the QCT and examined for correlations. Linear logistic regression was used to estimate bone density values base on HU.

Results A strong correlation was found between the HU measured in the CT and the bone density values $(r=0.894$, $p<0.001$ ), irrespective of patients' gender. We also found no correlation differences when the HU were measured at different levels. Bland-Altman plot demonstrated good agreement between the two measurements. The following formula was developed to estimate bone density values using HU: QCT-value $=0.71 \times \mathrm{HU}+13.82$.

Conclusions Bone density values correlate well to HU measured in contrast medium CT. Using simple formula, the bone density of a contrast medium CT of vertebral bodies can be estimated based on HU without additional examinations and unnecessary costs.
\end{abstract}

Keywords Hounsfield units · Bone mineral density $\cdot$ Quantitative computed tomography $\cdot$ Lumbar spine $\cdot$ Contrast medium

Fabian Buenger and Yasser Sakr have contributed equally to the work.

\section{Introduction}

Knowledge about bone density is essential for preoperative planning in patients with vertebral body fractures [1]. For optimum treatment results, one must not overlook an underlying osteoporosis. Due to demographic trends, an

1 Department of Neurosurgery, Jena University HospitalFriedrich Schiller University Jena, Jena, Germany

2 Department for Anaesthesiology and Intensive Care, Jena University Hospital-Friedrich Schiller University Jena, Jena, Germany

3 Department for Radiology, Jena University Hospital-Friedrich Schiller University Jena, Jena, Germany 
increasing proportion of elderly patients, who frequently suffer from reduced bone density are to be expected in the next years [2,3].

Quantitative computed tomography (QCT) has been routinely used to determine bone density. Recently, there have also been an increasing number of publications that determine bone density using the Hounsfield units (HU) in a native computed tomography $(\mathrm{CT})[4,5]$. In our own study, we were thus able to create a formula for patients with a native CT [6].

In the present study, we investigated the possible correlation between HU and QCT values in patients who received a thoraco-lumbar CT with contrast medium. Further, we aimed at developing a formula to calculate bone density based on the contrast medium computed tomography alone, thus possibly rendering additional QCTs redundant.

\section{Materials and methods}

We performed a retrospective data analysis of 98 vertebral bodies of 35 trauma patients who received contrast medium CT of the thoraco-lumbar spine and QCT of the lumbar spine in our department between 1 January 2015 and 15 February 2019.

All data were collected and processed using IBM SPSS statistical program for Windows (version 23; SPSS, Inc., Chicago, IL, USA). The following factors were determined: age, sex, mean values of Hounsfield units, mean values of
QCT, date of each examination. The study was approved by our local ethics committee.

\section{Inclusion and exclusion criteria}

All patients who received CT with contrast medium including the lumbar spine as part of a polytrauma CT scan were included. The axial, coronal and sagittal planes of the vertebral bodies had to have been reconstructed. QCT had to have been performed within 1 month prior or after. HU were measured in contrast medium CT from the 11th thoracic vertebral body to the 4th lumbar vertebral body and compared with the corresponding values from QCT of the associated vertebral bodies.

If pathologies, e.g. tumour, haemangioma, fractures, implants, cement augmented vertebral bodies, spondylodiscitis or osteochondrosis were detected in the $\mathrm{CT}$, the respective vertebral bodies were not used for the measurements.

\section{Measurement of the Hounsfield units}

Measurements were performed using CT (General Electric Medical Systems, USA, Revolution) in axial, sagittal, and coronal planes. The measurement field was chosen to be as large as possible, leaving out the cortex as described previously [6] (Fig. 1).

HU were determined with the software CernerSkyVue PACS Image Viewer (Cerner Deutschland GmbH), using the "Mean" value for further calculation. The mean value was determined from each plane and the mean HU was

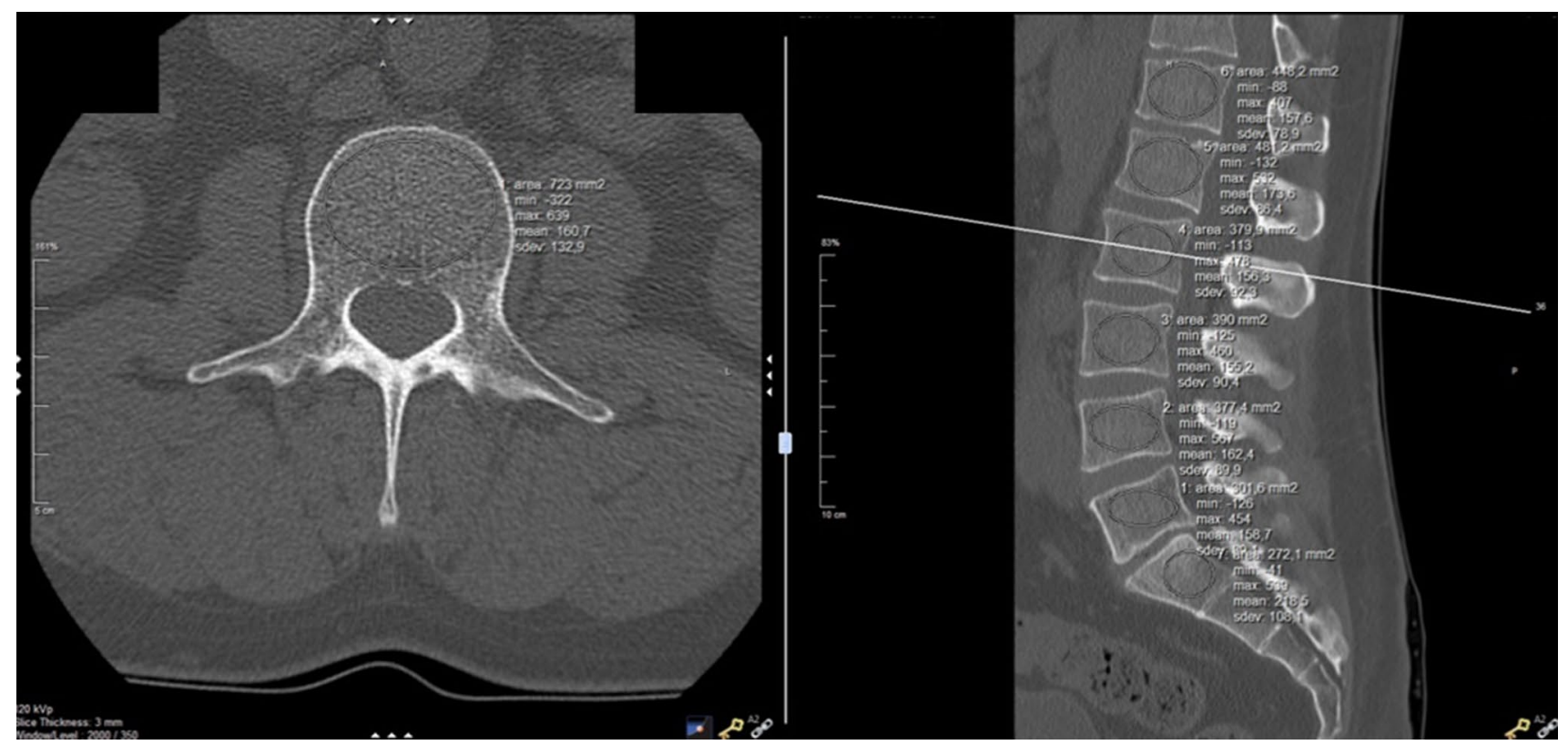

Fig. 1 Computed tomography of the spine with axial (left), sagittal (right) measurement of the Hounsfield units using an elliptical measurement field in the center of the vertebral bodies 
calculated from this. The measurements were always taken by the same physician.

\section{Measurement of the quantitative computed tomography values}

QCT values were measured on a CT scanner (General Electric Medical Systems, USA, Revolution EVO). The calibration phantom consisted of $\mathrm{K}_{2} \mathrm{HPO}_{4}$ and was placed under the patient. Bone density was determined using bone mineral densitometry software (QCT-Pro ${ }^{\mathrm{TM}}$, version 3.1, Mindways Software).

\section{Statistics}

Categorical data are given as number ( $n$ ) and percent (\%) and continuous data as mean with standard deviation (SD) or median with interquartile range (IQ).

Pearson's correlation coefficient or Spearman-Rho was used to use the correlation of 2 variables. A value of $r=1$ indicated that there was a linear relationship. A value of $r=-1$ showed an inverse correlation of the variables. A $t$ test was used to test whether the determined correlation coefficients differ from zero. Bland-Altman plot was used to assess the agreement between measurements. Linear logistic regression was used to estimate QCT values from HU units. A significance was evaluated as a value below $5 \%$.

\section{Results}

At the time of examination, the median age of the patients was 62 years (IQ: 53-74). Measurements were taken on 71 vertebral bodies of male (72.4\%) and 27 vertebral bodies of female patients $(27.6 \%)$. The median interval between contrast medium CT and QCT examination was 3 days (IQ: $1-7)$. The 2 nd lumbar was the most frequently examined vertebra with 30 measurements. Details are provided in Table 1.

The median $\mathrm{HU}$ of all vertebral bodies was 131.53 (IQ: 98.4-164.38), and $103.5 \mathrm{mg} / \mathrm{cm}^{3} \mathrm{~K}_{2} \mathrm{HPO}_{4}$ (IQ: 85.9-128.9) for QCT, respectively.

In the overall comparison, a significant correlation between the HU and the QCT values was found with a Pearson correlation coefficient $r=0.894(p<0.001)$ (Fig. 2), and this correlation was consistent in both, male $(r=0.904$, $p<0.001)$ and female patients $(r=0.887, p<0.001)$. Table 2 details correlation coefficients for each vertebral body, and Fig. 3 demonstrates good agreement between the measurements.
Table 1 QCT-values and the corresponding Hounsfield units according to the different vertebras

\begin{tabular}{lccc}
\hline & Count & QCT-values $^{\mathrm{a}}$ & \multicolumn{1}{l}{ Hounsfield units } \\
\hline Th 11 & 5 & $105.8(76.7-111.9)$ & $114(105.3-151.2)$ \\
Th 12 & 5 & $101.8(82.9-134.7)$ & $119(109.4-176.2)$ \\
L 1 & 18 & $115.6(80-133)$ & $157.5(95.2-176.6)$ \\
L 2 & 30 & $99.1(57.6-135.2)$ & $134.2(98.7-165.9)$ \\
L 3 & 25 & $102.3(60.2-124)$ & $131.6(93.3-164.3)$ \\
L 4 & 15 & $96.3(67.8-132.4)$ & $107.7(94.6-107.7)$ \\
All levels & 98 & $103.5(85.9-128.9)$ & $131.5(98.4-164.4)$ \\
\hline
\end{tabular}

Values are presented as median (25-75\% interquartile range)

$L$ lumbar, $T h$ thoracic

${ }^{\mathrm{a}} \mathrm{mg} / \mathrm{cm}^{3} \mathrm{~K}_{2} \mathrm{HPO}_{4}$

\section{Establishing a conversion formula}

Using a logistic linear regression, a following formula was established to approximate bone density values:

QCT-value $=0.71 \times \mathrm{HU}+13.82$.

\section{Discussion}

The aim of the present study was to examine whether QCT values of vertebral bodies correlate with $\mathrm{HU}$ of a contrast medium $\mathrm{CT}$ in the thoraco-lumbar spine, to solely rely on contrast medium CT to determine the bone mineral density and to refrain from additional examinations, thus reducing radiation dose.

In our study, we found a strong correlation between $\mathrm{HU}$ and QCT values with $r=0.894$.

Several papers discussing this topic have been published so far [7-10]. However, to the best of our knowledge, our series represents the largest series of vertebrae examined in patients without malignant disease. In most previous studies, no contrast-enhanced CT was used to compare HU with QCT.

In a previous study, Baum et al. examined 15 postmenopausal women who had a QCT and a contrast medium CT [7]. They, too, found a significant positive correlation with a $r=0.914$.

However, the duration between CT and QCT significantly longer with up to 3 months in their study. In the meantime, changes in bone density may have occurred due to various causes (cortisone therapy, progressive osteoporosis, and advanced tumour disease). To avoid these confounding factors, we only included patients in our study with a very short interval between examinations. The median time between $\mathrm{CT}$ and QCT was only 3 days in our cohort.

In another study by Bauer et al. 40 again postmenopausal women were examined with QCT and contrast CT of 
Fig. 2 Scatter plot showing quantitative computed tomography values in relation to the mean values of Hounsfield units from the 11th thoracic vertebral body to the 4 th lumbar vertebral body, $n=98$, correlation coefficient $r=0.89$

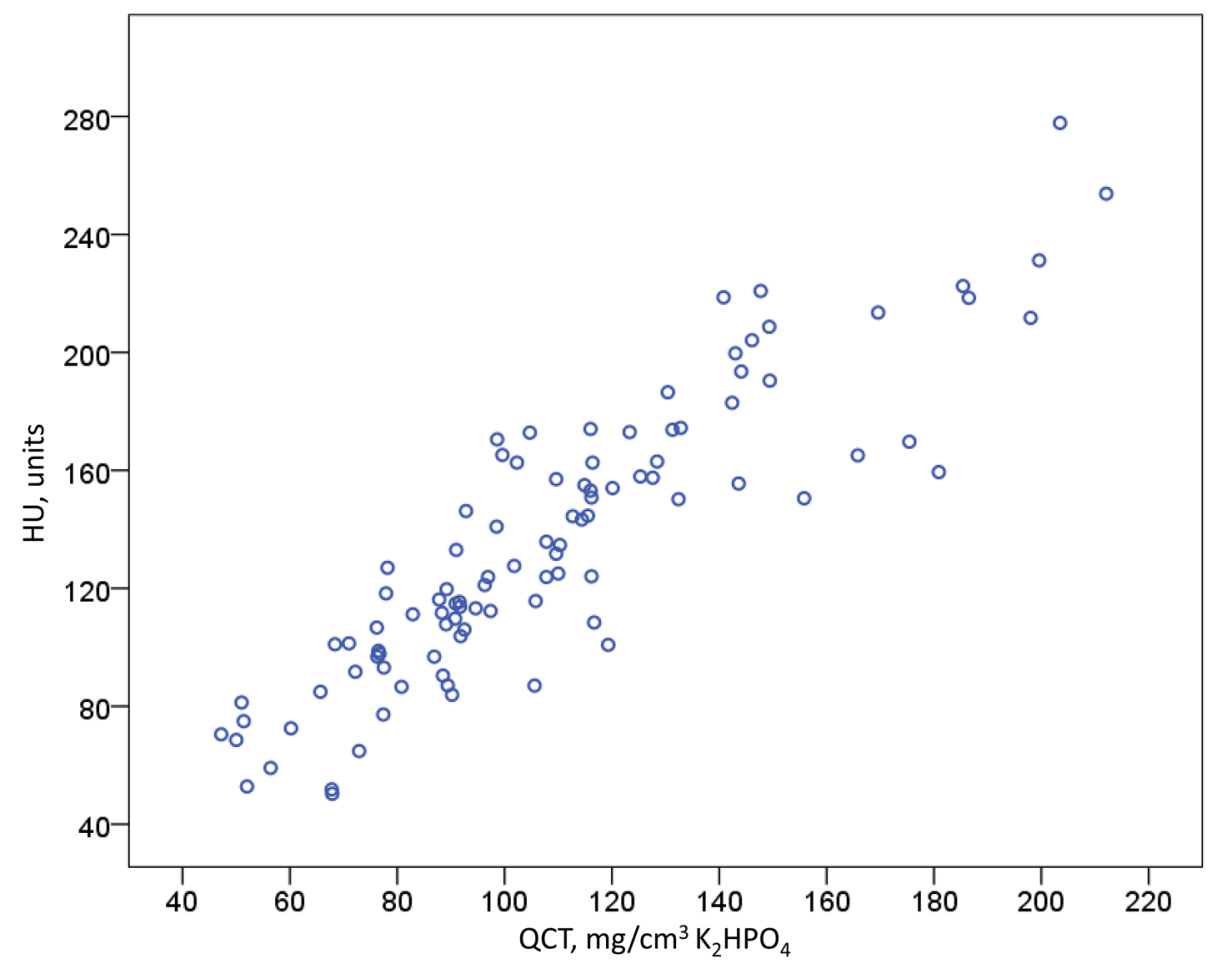

Table 2 Correlation of the mean values of contrast medium computed tomography and the values of quantitative computed tomography in the individual vertebral bodies

\begin{tabular}{lclr}
\hline & Count & $\begin{array}{l}\text { Correlation coeffi- } \\
\text { cient }(r)^{\mathrm{a}}\end{array}$ & $p$ value \\
\hline Th 11 & 5 & 0.823 & 0.087 \\
Th 12 & 5 & 0.900 & 0.087 \\
L 1 & 18 & 0.748 & $<0.001$ \\
L 2 & 30 & 0.902 & $<0.001$ \\
L 3 & 25 & 0.893 & $<0.001$ \\
L 4 & 15 & 0.943 & $<0.001$ \\
All levels & 98 & 0.894 & $<0.001$ \\
\hline
\end{tabular}

$L$ lumbar, $T h$ thoracic

${ }^{\text {a}}$ Pearson or Spearman-Rho, as appropriate

the abdomen [8]. Here, too, a positive linear correlation with a $r=0.98$ could be determined. Yet, most patients here received abdominal contrast-enhanced CT to screen for malignant disease, and many suffered from colorectal cancers. Since cancer can also lead to changes in bone density, we chose to analyse patients suffering from trauma and excluded cancer patients.

There are other studies that address this issue. However, in most cases, no contrast medium was given, so these studies are not comparable with our study. The administration of the contrast medium can significantly influence the HU in the CT due to the blood flow, the time of contrast medium administration and the respective tissue. This is often more pronounced in younger patients than in older patients [11, 12]. For example, the contrast medium signal in the area of the Batson venous plexus of the vertebra leads to higher Hounsfield units than in patients who have not received a contrast medium. For this reason, the use of a formula based on a native computed tomography is not transferable to Hounsfield units from contrast agent computed tomographies. In our measurements, we have only included patients who have had a contrast medium CT.

Table 3 lists possible formulas for calculating the bone density value from the various studies as well as our own data. Overall, the studies listed showed similarly high correlations, but the formulas differed by several percentage points in some cases.

One possible reason could be that only smaller numbers of cases were described in the available studies. In addition, the HU were not determined in multiple planes [8].

It could be possible that the Hounsfield units in contrast medium CT in a single plane do not represent the bone quality of the entire vertebra, as anatomical variants, bone canals or degenerative changes can lead to a deviation in the values. In our work, however, we measured in axial, coronal and sagittal planes.

Deviations in the QCT values may occur due to variations in the position of the patient on the CT table or variability related to different measuring surfaces. This may account for up to 5-15\% fluctuations in the measured values with a coefficient of variation of $1.3-1.7 \%$ [4, 5, $7,13]$. To minimise these deviations and to achieve valid 
Fig. 3 Bland-Altman plot demonstrating the difference between the QTC values and HU: mean: - 25.11; standard deviation 21.32 $(1.96 \times \mathrm{SD}=41.79$, upper limit $=16.68$, lower limit: $-66.9)$

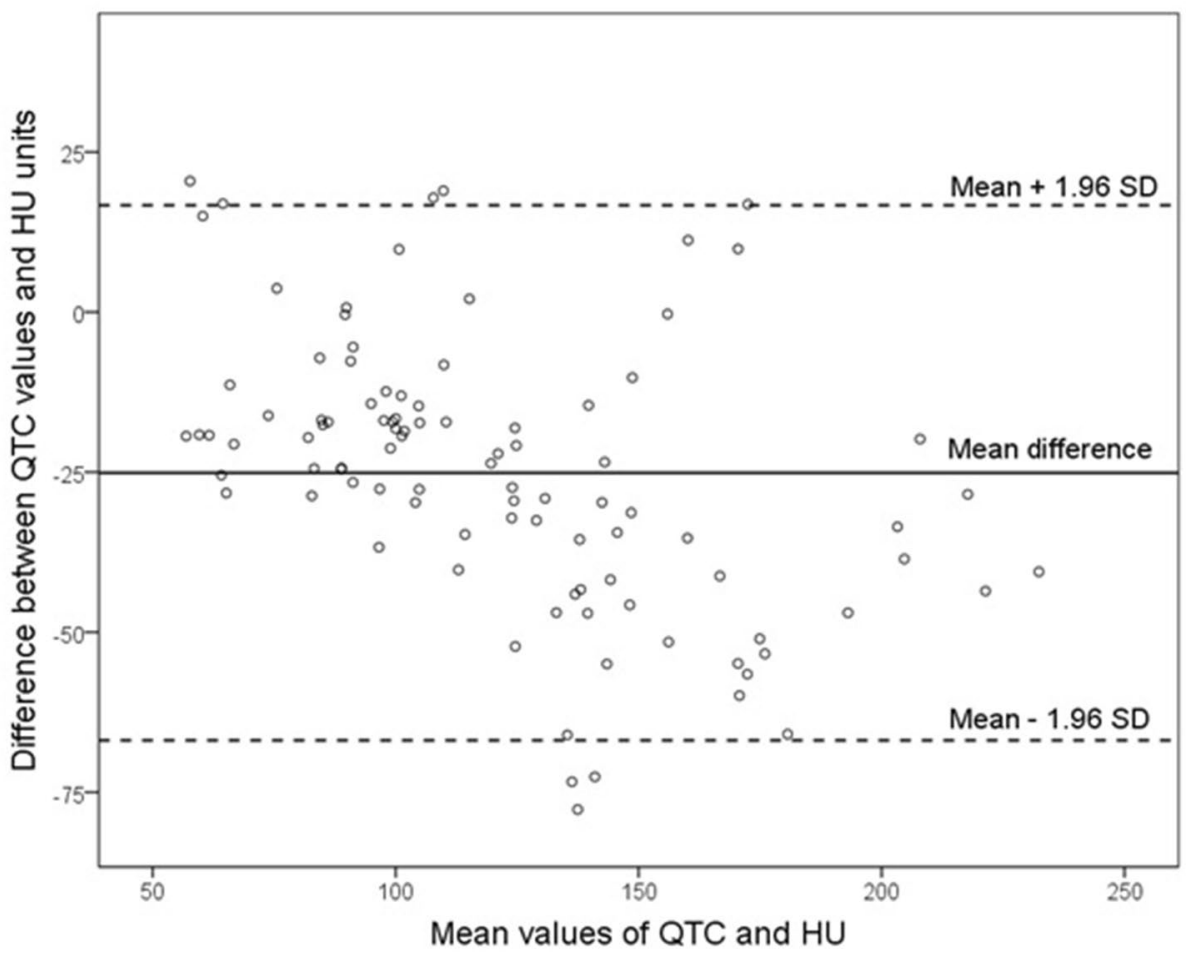

\section{Limitations}

Table 3 Overview of the different conversion formulas of different authors

\begin{tabular}{ll}
\hline Authors, year & Formula \\
\hline Baum et al. 2012 [7] & QCT-value $=0.695 \times \mathrm{HU}-7.9$ \\
Bauer et al. 2007 [8] & QCT-value $=0.96 \times \mathrm{HU}-20.9$ \\
Papadakis et al. 2009 [10] & QCT-value $=0.78 \times \mathrm{HU}+10.13$ \\
Our data & QCT-value $=0.71 \times \mathrm{HU}+13.82$ \\
\hline
\end{tabular}

values, only trained personnel performed measurements in our study in a standardized position using the same technical setting $[2,4,8,14]$.

All these factors can lead to deviations and thus to a low correlation coefficient.

As consequence for daily practice, we use these formula for patients who should operate with screw implantation in the spine. The American College Radiology defines osteoporosis in QCT with a BMD below $80 \mathrm{mg} /$ $\mathrm{cm}^{3}$, osteopenia is indicated with a BMD between 80 and $120 \mathrm{mg} / \mathrm{cm}^{3}$ and a value above $120 \mathrm{mg} / \mathrm{cm}^{3}$ describes a normal bone density [15]. In our clinic, we use perforated screws in patients with a bone density below $120 \mathrm{mg} / \mathrm{cm}^{3}$, so that intraoperatively, depending on the screw traction, it can be decided whether the screw should be additionally augmented with cement.
A limitation of our study is its retrospective and monocentric nature. A multicentre study with other CT scanners and protocols would be necessary to strengthen our findings.

All examinations were performed in the context of trauma, but the vertebral bodies involved in the trauma were excluded. However, the patients were not completely healthy.

\section{Conclusion}

Bone density values correlate well to HU measured in contrast medium CT. Using simple formula, the bone density of a contrast medium CT of vertebral bodies can be estimated based on $\mathrm{HU}$ without additional examinations and unnecessary costs.

Author contributions All individuals listed as authors have substantially contributed to the design, execution, and analysis of the study. Prior to its submission, all authors have reviewed and confirmed the accuracy of the whole manuscript.

Funding Open Access funding enabled and organized by Projekt DEAL. Not applicable. 


\section{Declarations}

Conflict of interest The authors declare that they have no conflict of interest.

Ethical approval The study was approved by the local ethic committee.

Informed consent Not necessary.

Open Access This article is licensed under a Creative Commons Attribution 4.0 International License, which permits use, sharing, adaptation, distribution and reproduction in any medium or format, as long as you give appropriate credit to the original author(s) and the source, provide a link to the Creative Commons licence, and indicate if changes were made. The images or other third party material in this article are included in the article's Creative Commons licence, unless indicated otherwise in a credit line to the material. If material is not included in the article's Creative Commons licence and your intended use is not permitted by statutory regulation or exceeds the permitted use, you will need to obtain permission directly from the copyright holder. To view a copy of this licence, visit http://creativecommons.org/licenses/by/4.0/.

\section{References}

1. Ullrich BW, Schenk P, Spiegl UJ, Mendel T, Hofmann GO (2018) Hounsfield units as predictor for cage subsidence and loss of reduction: following posterior-anterior stabilization in thoracolumbar spine fractures. Eur Spine J 27:3034-3042. https://doi. org/10.1007/s00586-018-5792-9

2. Schwaiger BJ, Gersing AS, Baum T, Noel PB, Zimmer C, Bauer JS (2014) Bone mineral density values derived from routine lumbar spine multidetector row CT predict osteoporotic vertebral fractures and screw loosening. AJNR Am J Neuroradiol 35:1628 1633. https://doi.org/10.3174/ajnr.A3893

3. Reginster JY, Burlet N (2006) Osteoporosis: a still increasing prevalence. Bone 38:S4-9. https://doi.org/10.1016/j.bone.2005. 11.024

4. Adams JE (2009) Quantitative computed tomography. Eur J Radiol 71:415-424. https://doi.org/10.1016/j.ejrad.2009.04.074

5. Guglielmi G, Lang TF (2002) Quantitative computed tomography. Semin Musculoskelet Radiol 6:219-227. https://doi.org/10. 1055/s-2002-36719

6. Buenger F, Eckardt N, Sakr Y, Senft C, Schwarz F (2021) Correlation of bone density values of quantitative computed tomography and Hounsfield units measured in native computed tomography in 902 vertebral bodies. World Neurosurg. https://doi.org/10.1016/j. wneu.2021.04.093

7. Baum T, Muller D, Dobritz M, Wolf P, Rummeny EJ, Link TM, Bauer JS (2012) Converted lumbar BMD values derived from sagittal reformations of contrast-enhanced MDCT predict incidental osteoporotic vertebral fractures. Calcif Tissue Int 90:481-487. https://doi.org/10.1007/s00223-012-9596-3

8. Bauer JS, Henning TD, Mueller D, Lu Y, Majumdar S, Link TM (2007) Volumetric quantitative CT of the spine and hip derived from contrast-enhanced MDCT: conversion factors. AJR Am J Roentgenol 188:1294-1301. https://doi.org/10.2214/AJR.06.1006

9. Mueller DK, Kutscherenko A, Bartel H, Vlassenbroek A, Ourednicek P, Erckenbrecht J (2011) Phantom-less QCT BMD system as screening tool for osteoporosis without additional radiation. Eur J Radiol 79:375-381. https://doi.org/10.1016/j.ejrad.2010.02.008

10. Papadakis AE, Karantanas AH, Papadokostakis G, Petinellis E, Damilakis J (2009) Can abdominal multi-detector CT diagnose spinal osteoporosis? Eur Radiol 19:172-176. https://doi.org/10. 1007/s00330-008-1099-2

11. Center JR, Nguyen TV, Schneider D, Sambrook PN, Eisman JA (1999) Mortality after all major types of osteoporotic fracture in men and women: an observational study. Lancet 353:878-882. https://doi.org/10.1016/S0140-6736(98)09075-8

12. Chrischilles E, Shireman T, Wallace R (1994) Costs and health effects of osteoporotic fractures. Bone 15:377-386. https://doi. org/10.1016/8756-3282(94)90813-3

13. Johnson TR, Krauss B, Sedlmair M, Grasruck M, Bruder H, Morhard D, Fink C, Weckbach S, Lenhard M, Schmidt B, Flohr T, Reiser MF, Becker CR (2007) Material differentiation by dual energy CT: initial experience. Eur Radiol 17:1510-1517. https:// doi.org/10.1007/s00330-006-0517-6

14. Coppenrath E, Schmid C, Brandl R, Szeimies U, Hahn K (2001) Spiral CT of the abdomen: weight-adjusted dose reduction. Rofo 173:52-56. https://doi.org/10.1055/s-2001-10226

15. Kanis JA, Cooper C, Rizzoli R, Reginster JY, Scientific Advisory Board of the European Society for C, Economic Aspects of O, the Committees of Scientific A, National Societies of the International Osteoporosis F (2019) European guidance for the diagnosis and management of osteoporosis in postmenopausal women. Osteoporos Int 30(1):3-44

Publisher's Note Springer Nature remains neutral with regard to jurisdictional claims in published maps and institutional affiliations. 